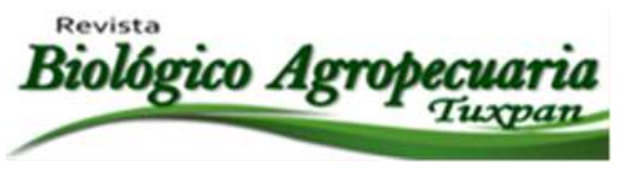

\title{
Salsa a base de zarzamora (Rubus fruticosus) como potencial alimento funcional
}

Blackberry sauce (Rubus fruticosus) like a potential functional food.

Ceballos-Luna Oscar ${ }^{1}$, Morales-Sotelo Rogelioํㅜ, López-Márquez Alex Amir ${ }^{1}$, Grapain-López Orquídea ${ }^{1}$, García-

Gutiérrez Emmanuel ${ }^{1}$, Sedeño-Mota Juan Carlos ${ }^{1}$, Álvarez Campos Edith ${ }^{2}$, Lozada García José Armando ${ }^{1 凶}$

${ }^{1}$ Facultad de Biología Xalapa, Universidad Veracruzana. Circuito Universitario Gonzalo Aguirre Beltrán s/n, Col. Zona Universitaria, Xalapa, Veracruz C.P. 91000

${ }^{2}$ Unidad de Capacitación para el Desarrollo Rural No. 2 Tlanalapa s/n Col. Los Carriles, Coatepec, Veracruz, México. C.P. 91500

${ }^{\bowtie}$ Autor de correspondencia: alozada@uv.mx

Recibido: 13/07/2018
Aceptado: $12 / 09 / 2018$

\section{RESUMEN}

La zarzamora (Rubus fruticosus) es una rosácea comestible cultivada en la microrregión de Coyopolan municipio de Ixhuacán de los Reyes, Veracruz, México. Esta fruta es importante en esta localidad ya que es utilizada en la elaboración de una variedad de productos artesanales, entre los que destacan el licor de zarzamora y mermelada. Algunos de estos productos además de ser un aporte económico para los habitantes, proporcionan un beneficio a la salud por sus componentes como los compuestos fenólicos que actúan como antioxidantes naturales. En este sentido el presente trabajo tuvo como objetivo elaborar una salsa como potencial alimento funcional teniendo como ingrediente principal la zarzamora y una variedad local de chile, llamado "tabaquero"; a estas salsas se les evaluó su calidad nutricional, la presencia de microorganismos a través de pruebas como coliformes totales, conteo de bacterias aerobias en placa y conteo de mohos y levaduras en alimentos, posteriormente se cuantificaronlos flavonoides, fenoles y antocianinas totales, así como también su actividad antioxidante. Como resultado se obtuvieron tres tipos de salsa, en las que se determinó que no hay presencia de microorganismos, las pruebas de calidad nutrimental indicaron que tienen buenos valores y respecto a la funcionalidad se observó que, en comparación con el fruto fresco se mantuvo la actividad antioxidante aún después del proceso. Por lo que concluimos que los productos cuentan con valores apropiados para considerarlos como potenciales alimentos funcionales.

Palabras clave: alimento funcional, antioxidante, zarzamora, flavonoides.

\begin{abstract}
The blackberry (Rubus fruticosus) is an edible rosacea grown in the microregion of Coyopolan at Ixhuacán de los Reyes, Veracruz, México. This one is important in the region since it is used in the
\end{abstract}


elaboration of a big variety of products, among which stand out the liquor of blackberry and jam. Some products in addition to be the economic sustenance for the habitants of the region, has health benefits from some components such as phenolic compounds that act as natural antioxidants. In this job has the objective of develop a sauce as a functional food having as principal ingredient blackberrys, also using a local variety of chili, called "tabaquero chili", and this sauce was evaluated their nutritional quality, the presence of total coliforms, quantification of flavonoids, phenols, anthocyanins and antioxidant activity. Three types of sauce were obtained, from which it was determined that there is not presence of total coliforms in the sauces, the nutritional quality tests indicate that they have a low contribution in high density lipids (bad cholesterol) and the functionality is observed that the product and the three sauces in comparison maintains an activity antioxidant. So we conclude that the product has the appropriate values to consider it a functional food.

Keywords: functional food, antioxidant, blackberry, flavonoids.

\section{INTRODUCCIÓN}

La tendencia mundial busca en los alimentos la contribución de ciertos bioactivos que sean capaces de ejercer alguna acción beneficiosa al consumidor; estos aspectos son los que se buscan en los llamados "alimentos funcionales", productos nutracéuticos o farmalimentos (Bello, 1995; Aranceta et al., 2003). En los últimos años el interés por estos productos ha incrementado su demanda, principalmente por las propiedades antioxidantes con las que cuentan, entre éstos se encuentran los frutos rojos o "berrys" (Coronado et al., 2014).

Uno de los frutos rojos que contienen altos niveles de antioxidantes, flavonoides, antocianinas y taninos son las especies de Rubus (De Carvalho et al., 2010), es por ello que el objetivo del presente estudio fue incorporar estos metabolitos secundarios con actividad biológica en productos innovadores con características de alimento funcional, con la finalidad de apoyar a los productores de la microrregión de Coyopolan, municipio de Ixhuacán de los Reyes, Veracruz, México.

\section{MATERIALES Y MÉTODOS}

Se elaboraron tres salsas a base de Zarzamora (Rubus fruticosus) y una variedad de chile local conocida como "Tabaquero", la primera de estas era dulce, la segunda salada y la tercera salada condimentada con hoja de acuyo (Piper auritum). Todas las salsas fueron pasteurizadas y guardadas al vacío.

Una vez obtenida la salsa base que contenía 30 gr de azúcar y 20 gr de sal, se pasteurizó y se dividió en tres porciones iguales, las cuales correspondierona cada uno de los tres tipos de salsas, la primera porción se le agregó más azúcar adicionándole 110 gr, a la segunda se le agregó 10 gr de sal y finalmente a la tercera se le incorporó un triturado de una hoja asada de Piper auritum pesando aproximadamente $25 \mathrm{gr}$, además de 5 gr de sal.

\section{Calidad Alimenticia y Microbiológica}

Se evaluaron parámetros de calidad nutrimental con pruebas como contenido de grasas, humedad, pH, acidez, cenizas y azúcares bajo los estándares internacionles de la AOAC (Asociación de Químicos Analíticos Oficiales, 2015). La cantidad de proteína total se determinó 
mediante el método de Biuret (Coutiño et al., 2015 Horwitz, 1990). Para el caso de los parámetros microbiológicos se realizazon con base en las Normas Oficiales Mexicanas:NOM113-SSA1-1994, NOM-111-SSA1-1994 y NOM-092-SSA1-1994.

Determinacion de flavonoides, fenoles, antocianinas totales y actividad antioxidante

Se realizaron pruebas para determinar el contenido de estos compuestos en frutos frescos y en las salsas, mediante técnicas espectrofotométricas como reducción de $\mathrm{AlCl} 3$, Folin \& Ciocalteun y pH diferencial (PyrkoszBiardzka et al., 2014, Palomino et al., 2009). La actividad antioxidante se calculó mediante el método de reducción de Molibdato (Moncada et al., 2012).

\section{RESULTADOS Y DISCUSIÓN}

Como se observa en la tabla 1, las salsas mostraron tener baja cantidad de grasa por cada muestra de 100 gr, la humedad contenida es de aproximadamente 50 a $60 \%$, las proteínas totales oscilan entre el 7 y 9\%. Los azúcares totales variaron en las salsas, siendo la salsa dulce la de mayor cantidad en porcentaje. En lo que respecta a la acidez del producto, fue menor al $1 \%$, lo que indica que es ligeramente acida al paladar. Finalmente, para el caso del pH, el promedio fue de tres.

Tabla 1. Información nutrimental y microbiológica de las salsas de Zarzamora.

$\begin{array}{lccc} & \text { Dulce } & \text { Salada } & \text { Salsa con Acuyo } \\ \text { Grasas } & 0.4313 \mathrm{gr} & 0.1896 \mathrm{gr} & 0.0993 \mathrm{gr} \\ \text { Humedad } & 59 \% & 51 \% & 49 \% \\ \text { pH } & 3.02 & 2.8 & 2.89 \\ \text { Acidez } & 1 \% & 1 \% & 1 \% \\ \text { Cenizas } & 38 \% & 18 \% & 15 \% \\ \text { Proteínas } & 8 \% & 9 \% & 7 \% \\ \text { Azúcares } & 50 \% & 36 \% & 40 \% \\ \text { NOM-092-SSA1-1994 } & 2 \text { UFC } & 2 \text { UFC } & 5 \text { UFC } \\ \text { NOM-113-SSA1-1994 } & 0 \text { UFC } & 0 \text { UFC } & 0 \text { UFC } \\ \text { NOM-111-SSA1-1994 } & 0 \text { UFM } & 0 \text { UFM } & 0 \text { UFM }\end{array}$

En cuanto al contenido de microorganismos anaerobios se encontró mayor presencia en la salsa de acuyo con un promedio de 5 UFC, de acuerdo a la NOM-092-SSA1-1994, estos se encuentran dentro de los valores de aceptación, para coliformes totales no hubo presencia a las $24 \mathrm{~h}$ de incubación, en el caso de mohos y levaduras se encontraron por debajo de los parámetros mínimos permicibles, por lo cual se 
consideran libres de estos microorganismos (NOM-092, NOM-111, NOM-113, 1994).

Actividad antioxidante y compuestos fenólicos

En cuanto a los flavonoides totales, el fruto fresco y la salsa salada, presentaron una concentración promedio de $0.41 \mathrm{mg}$ EQ (Equivalentes de Quercetina), lo cual sugiere que éstos permanecen después del proceso de elaboración. La salsa dulce presentó mayor contenido de flavonoides totales, y triplicó el contenido de estos polifenoles en comparación con el fruto fresco. La presencia de flavonoides como la quercetina sugirió que puede tener beneficios a la salud previniendo diversos tipos de enfermedades crónico-degenerativas por su actividad antioxidante (Knekt et al., 2002; Geleijnse et al., 2002).
En cuanto a las antocianinas totales (fruto fresco, salsas salada, dulce y con acuyo) presentaron un bajo contenido entre 0.004-0.009 de mg equivalentes Cianidina3-O-glucósido. Los fenoles fueron los únicos compuestos que no se pudieron determinar en ninguna de las muestras, esto se debe a que se encuentran en cantidades mínimas.

Respecto a la actividad antioxidante que hayentre el fruto fresco, las salsas dulce y salada, los resultados muestran que esta característica se mantiene aún después del proceso de elaboración (Tabla 2). Por otra parte, cabe resaltar que la salsa con acuyo tuvo mayor actividad antioxidante, esto puede deberse a que esta planta aporta moléculas con esta característica, mostrando un alto contenido en compuestos fenólicos (García et al., 2002).

Tabla 2. Contenido de compuestos fenólicos y actividad antioxidante.

Compuestos Fenólicos cuantificados /Prueba

\begin{tabular}{|c|c|c|c|c|}
\hline Muestra & $\begin{array}{l}\text { Flavonoides } \\
\text { Reducción de ClAl3 } \\
\text { (mg EQ) }\end{array}$ & $\begin{array}{l}\text { Fenoles } \\
\text { Folin\&Ciocalteun } \\
(\text { mg EAT) }\end{array}$ & $\begin{array}{l}\text { Antocianinas } \\
\text { pH diferencial } \\
\text { (mg = cyanidin 3-O- } \\
\text { glucoside }\end{array}$ & $\begin{array}{l}\text { Actividad antioxidante } \\
\text { (reducción de } \\
\text { molibdato) }\end{array}$ \\
\hline Fruto fresco & 0.36 & 0 & 0.004 & 1.62 \\
\hline Salsa dulce & 1.31 & 0 & 0.009 & 1.64 \\
\hline Salsa salada & .0 .47 & 0 & 0.009 & 1.39 \\
\hline $\begin{array}{l}\text { Salsa con } \\
\text { acuyo }\end{array}$ & 0.21 & 0 & 0.006 & 5.59 \\
\hline
\end{tabular}

EQ: Equivalentes a Quercetina, EAT= Equivalentes a Ácido Tánico

\section{CONCLUSIÓN}

Se puede inferir que las salsas son un producto innovador ya que pueden ser consideradas un potencial alimento funcional, además de contar con valores nutrimentales aceptables y estándares microbiológicos dentro de las normas oficiales mexicanas. Por otra parte se le incorporaron compuestos fenólicos de la 
zarzamora, como son los flavonoides y las antocianinas los cuales son benefiosos para la salud humana, además de que muestran actividad antioxidante significativa, en especial la salsa que contiene acuyo.

\section{LITERATURA CITADA}

Aranceta, J. Y Serra, L. 2003. Guía de alimentos funcionales. Sociedad Española de Nutrición Comunitaria (SENC). 105.

Bello, J. 1995. Los alimentos funcionales o nutracéuticos. Nueva gama de productos en la industria alimentaria. Alimentaria 265: 25-29.

Coronado, G. M. A., García, P. M., Santiago, H. V., García, P. M., Santiago, H. V. G., Córdova,Y. A. y Vásquez, N. R. 2014. La zarzamora, un mercado potencial para los productores agropecuarios de la Sierra de Sonora. Sociedad Mexicana de Administración Agropecuaria A.C. Torreón, México.784-794.

Coutiño, R., Fernández, S y Palmeros, B. 2015. Manual de la experiencia educativa biología celular. Universidad Veracruzana. 132. https://doi.org/10.25009/uv.2018.37

De Carvalho, T., Thomsen, M. R. y Clark, J. R. 2010. Commercial fresh blackberry shipping market growth and price trends in the United States. Small Fruit News.102: 11.

García, L., Rojo, D. M. D., García, L. V. G. y Hernández, M. A. 2002. Plantas con propiedades antiinflamatorias. Revista Cubana de Investigaciones Biomédicas 21(3): 214-216.

Geleijnse, J. M., Launer, L. J, Van der Kuip, D. A. M, Hofman, A. y Witteman, J. C. M.
2002. Inverse association of tea and flavonoid intakes with incident myocardial infection: the Rotterdam study. The American Journal of Clinical Nutrition 75, 880-886.

https://doi.org/10.1093/ajen/75.5.880

Horwitz, W.1990. Official methods of analysis Washington, DC: Association of Official Analytical Chemists. 54: 171.

Knet, P., Järvinen, R., Reunanen, A. y Maatela, J. 1996. Flavonoid intake and coronary mortality in Finland: A cohort Study. British. Medical Journal 312: 478-481. https://doi.org/10.1136/bmj.312.7029.47 8

Martínez, N. S. 2012. Producción de antocianinas por cultivo "in vitro" de Rubusa denotrichus Schltdl (zarzamora). Tesis de doctorado. División de estudios de posgrado. Facultad de ciencias biológicas. Universidad Autónoma de Nuevo León. Monterrey, Nuevo León, México. 85.

Moncada, M. V., Giraldo, A.M. y Landazuri, P. 2012. Actividad antioxidante de los extractos etanólicos y acuosos de la cáscara y la semilla de Annona muricata y de las hojas brownea ariza. Revista de la Asociación Colombiana de Ciencias Biológicas 24: 143-151.

Palomino, G. L. R., García, C. M. P., Gil, J. H., Rojano, G. B. A. y Durango, D. L. 2009. Determinación del contenido de fenoles y evaluación de la actividad antioxidante de propóleos recolectados en el departamento de Antioquia (Colombia). Vitae 16 (3): 388-395.

Secretaria de salud. Norma Oficial Mexicana NOM-092-SSA1-1994, bienes y servicios. Métodos para la cuenta de bacterias aerobias en placa. Recuperado de: 
http://www.ordenjuridico.gob.mx/Docu mentos/Federal/wo69532.pdf

Secretaria de salud. Norma Oficial Mexicana NOM-113-SSA1-1994, bienes y servicios. Método para la cuenta de microorganismos coliformes totales en placa. Recuperado de: http://www.dof.gob.mx/normasOficiales /3924/stps1/stps1.htm

Secretaria de salud. Norma Oficial Mexicana, NOM-111-SSA1-1994, bienes y servicios. Método para la cuenta de mohos y levaduras en alimentos. Recuperado de: http://www.cofepris.gob.mx/MJ/Docum ents/Normas/111ssa1.pdf

Pyrkosz-Biardzka, K., Kucharska, A. Z, SokółŁętowska, A., Strugala, P. y Gabrielska, J. 2014. A Comprehensive Study on Antioxidant Properties of Crude extracts from Fruits of Berberis vulgaris L., Cornus mas L. and Mahonia aquifolium. Polish Journal Food and Nutrition Science 64(2): 91-99.

https://doi.org/10.2478/v10222-012-009 $7-\mathrm{x}$

Copyright (c) 2018 Oscar Ceballos Luna, Rogelio Morales Sotelo, Alex Amir López Márquez, Orquidea Grapain López, Emm anuel G arcia Gutiérrez, Juan Carlos Sedeño Mota, Edith Álvarez Cam pos y José Armando L ozada Garcia

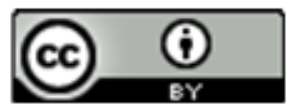

Este tex to está protegido por una licencia Creative Commons 4.0

Usted es libre para Compartir — copiar y redistribuir el material en cualquier medio o formato-y Adaptar el documento — remezclar, transformar y crear a partir del material- para cualquier propósito, incluso para fines com erciales, siempre que cumpla la condiciơn de:

Atribución: Usted debe dar crédito a la obra original de manera adecuada, proporcionar un enlace a la licencia, e in đicar si se han realizado cambios. Puede hacerlo en cualquier forma razonable, pero no de forma tal que sugiera que tiene el apoyo del licenciante o lo recibe por el uso que hace de la obra.

Resumendelicencia - Textocompletodelalicencia 